\title{
Tuna extract reduces serum uric acid in gout-free subjects with insignificantly high serum uric acid: A randomized controlled trial
}

\author{
DAIKI KUBOMURA, MASANORI YAMADA and AYANO MASUI \\ Yaizu Suisankagaku Industry Co., Ltd., Shizuoka 425-8570, Japan
}

Received March 21, 2016; Accepted May 30, 2016

DOI: 10.3892/br.2016.701

\begin{abstract}
Long-term reduction of serum urate levels is vital in the treatment of gout. However, it is difficult to convince gout-free individuals of the necessity of treatment as few appropriate over-the-counter remedies and dietary supplements are available. Therefore, the present study aimed to investigate the antihyperuricemic efficacy and safety of a tuna extract containing the imidazole compounds to evaluate its potential as a functional food ingredient. A randomized, 4-week, double-blind, placebo-controlled study was conducted. A total of 48 male gout-free subjects with insignificantly high serum uric acid were randomly assigned to low- and high-dose tuna extract groups or a placebo group. The efficacy of the extract was assessed by measuring serum uric acid levels. Furthermore, a safety assessment was performed by physical parameters, hematology, blood biochemistry and urinalysis. The results indicated that the uric acid level was decreased at week 4 during the intervention in the tuna extract groups (low and high dose, -0.23 and $-0.34 \mathrm{mg} / \mathrm{dl}$, respectively) compared to the placebo group $(-0.07 \mathrm{mg} / \mathrm{dl})$. At week 4 after the intervention, a significant reduction in uric acid levels $(-0.41 \mathrm{mg} / \mathrm{dl} ; \mathrm{P}<0.05)$ was observed in the high-dose tuna extract group compared with the placebo group $(+0.11 \mathrm{mg} / \mathrm{dl})$. No dose-related adverse events were observed during and following the intervention. Therefore, the present results suggest that oral administration of tuna extract containing the imidazole compounds has hypouricemic activity with no undesirable side effects.
\end{abstract}

\section{Introduction}

Gout is characterized by the recurrent attacks of acute inflammatory arthritis with marked suffering. Serum uric acid reduction is crucial as elevation of uric acid concentrations, the

Correspondence to: Dr Daiki Kubomura, Yaizu Suisankagaku Industry Co., Ltd., 5-8-13 Kogawa-Shimmachi, Shizuoka 425-8570, Japan

E-mail: kubomura@yskf.co.jp

Key words: dietary supplement, anserine, imidazolic peptide, uric acid, hyperuricemia cause of gout, may result in metabolic syndrome (1). However, the incidence of gout has rapidly increased over the last 30 years in Japan, corresponding with increases in meat, fish and alcohol consumption, which is a result of Westernization. Uric acid is the final oxidation product of purine metabolism in humans in the absence of the hepatic enzyme uricase. Increased production and/or decreased uric acid excretion elevated serum uric acid levels. The former is caused by an excessively purine-rich diet and purine metabolism overactivation, whereas the latter is caused by renal impairment and certain drugs. Hyperuricemia is diagnosed when serum uric acid levels exceed the limit of solubility $(7.0 \mathrm{mg} / \mathrm{dl})$, and increases the risk of monosodium urate or uric acid crystal deposition, which could result in acute gouty arthritis, gouty arthropathy, chronic tophaceous gout, uric acid urolithiasis or gouty nephropathy (2). In addition, hyperuricemia is considered as a risk factor for the incidence of cardiovascular diseases (3). Thus, long-term reduction of serum urate concentrations to subsaturation levels is vital in the treatment of gout (4).

Clinical management of serum uric acid levels often includes using a xanthine oxidase inhibitor (allopurinol) and uricosurics (probenecid and benzbromarone), which facilitate urinary excretion. However, their use can induce several adverse reactions, such as fever, skin rash, worsened renal function and Stevens-Johnson syndrome $(5,6)$. The risk-benefit balance for using such drugs among gout-free patients with hyperuricemia is not favorable according to the guidelines for the management of hyperuricemia and gout (7). Therefore, non-medication treatments, including a low-purine diet, exercise therapy and natural products, are recommended for gout-free individuals with insignificantly high serum uric acid. In particular, it is commonly assumed that regular ingestion of dietary supplements is easier in comparison to dietetics or exercise therapy for the individuals with insignificantly high serum uric acid and no gout pain. Although the majority of physicians have not regarded dietary supplements to be efficacious on hyperuricemia, the ingredients have been investigated for hypouricemic activity.

Imidazole compounds (L-histidine, anserine and carnosine) are found at high concentrations in the muscles and brains of vertebrates (8). Various studies have indicated that the imidazole compounds have pH-buffering (9) and antioxidant properties (10), which are attributable to their imidazole moiety. The imidazole compounds have been reported to affect the activities of enzymes involved in glycogenolysis (11) 
and gluconeogenesis (12). These properties suggest that the imidazole compounds may affect the $\mathrm{pH}$ of body fluids, renal functions and organic acid levels that affect uric acid reabsorption in the glycolytic pathway, resulting in decreased serum uric acid levels. In addition, an early investigation proposed a tuna extract containing the imidazole compounds as a hypouricemic agent in a preliminary clinical study (13). Therefore, the present study evaluated the efficacy and safety of the tuna extract containing a high concentration of the imidazole compounds on serum uric acid levels in subjects with insignificantly high serum uric acid.

\section{Materials and methods}

Subjects and eligibility. All the volunteers were recruited from the Mareesia Garden Clinic (Tokyo, Japan), and were Japanese male volunteers (aged 20-64 years) whose serum uric acid concentrations were $6.5-8.0 \mathrm{mg} / \mathrm{dl}$, the subject of lifestyle guidance, unless they had any complications. Women were excluded from the present study due to the small size of the sample population. The exclusion criteria were gout, serious nephropathy, hepatopathy, cardiac disease, anaphylaxis reaction to the test compound and other abnormalities (as deemed by the investigator). Furthermore, volunteers already receiving urate-lowering therapies, including drugs and dietary supplements, were excluded. The final exclusion criterion was participation in another clinical study at the beginning of the present study. In total, 48 subjects who fulfilled the eligibility criteria for the study agreed to participate. All 48 subjects were enrolled in the study prior to random allocation into the three groups.

Approval of the study was obtained from the institutional review board of the Mareesia Garden Clinic, according to an ethical principle and experimental plan based on the Helsinki Declaration and the Ethical Guidelines for Epidemiological Research by the Japanese Government. Prior to enrollment in the study, a complete explanation regarding the study objective and methodology was provided to the subjects by the doctor; and participants in the study provided written informed consent.

Study design. The study was performed according to a randomized, double-blind, placebo-controlled design. Following screening and enrollment, 48 subjects were assigned using a random number table to one of three groups: The placebo and the test supplement at low and high doses of tuna extract. The subjects in the test supplement groups were provided with hydroxypropyl methylcellulose capsules filled with a commercial tuna extract, Marine Active 10 (Yaizu Suisankagaku Industry Co., Ltd., Shizuoka, Japan). Marine Active 10 is produced by ion-exchange purification and spray drying from a hot water extract of skipjack and yellowfin tuna, without enzymatic hydrolysis. Dextrin was added as an excipient, and the purine base was reduced to the level of $0.01 \mathrm{~g} / 100 \mathrm{~g}$. Quantification of amino acids and histidine-containing dipeptides in this product was performed using an amino acid analyzer (L-8500A; Hitachi, Tokyo, Japan) and high-performance liquid chromatography (14). The subjects in the placebo group were provided with hydroxypropyl methylcellulose capsules filled with dextrin. The contents of
Table I. Daily intake of the placebo and the test supplement (6 capsules/day).

Test supplement dose, $\mathrm{mg}$

\begin{tabular}{lrrr} 
& Placebo, mg & Low & High \\
\cline { 3 - 4 } Tuna extract & 0.0 & 238.6 & 477.1 \\
Anserine & 0.0 & 25.0 & 50.0 \\
Carnosine & 0.0 & 2.2 & 4.4 \\
Histidine & 0.0 & 16.9 & 33.9 \\
Other amino acids & 0.0 & 4.5 & 9.1 \\
Other proteins and peptides & 0.0 & 11.9 & 23.9 \\
Dextrin & 0.0 & 167.0 & 334.0 \\
Purine base & 0.0 & 0.0 & 0.0 \\
Dextrin & $1,250.9$ & $1,012.3$ & 773.7 \\
Calcium stearate & 65.8 & 65.8 & 65.8 \\
Total & $1,316.7$ & $1,078.1$ & 839.5 \\
\end{tabular}

Amounts of other proteins and peptides were calculated to correspond with the difference in the amounts of total amino acids and free amino acids. Dextrin and calcium stearate were added as excipients.

the placebo and the test supplement (6 capsules/day) are shown in Table I.

The subjects were treated with 2 capsules containing the placebo or the test supplement after each meal for 4 weeks. They visited the clinic at 7 days before the intervention, at weeks 2 and 4 during the intervention, and at week 2 after the intervention for follow-up assessment. The subjects were prohibited from drinking alcohol for 3 days and had to fast for $8 \mathrm{~h}$ before blood collection. During the visiting days, $24 \mathrm{ml}$ of blood was collected from each subject by venipuncture, which was then subjected to serum uric acid analysis (primary endpoint) and safety monitoring; this involved complete blood counts, qualitative urinalysis and measurement of body weight, body mass index, blood pressure, cardiac rate, total protein, albumin, albumin/globulin ratio, lactate dehydrogenase, alkaline phosphatase, aspartate aminotransferase, alanine aminotransferase, $\gamma$-glutamyl transpeptidase, creatinine, blood urea nitrogen, triglycerides, total cholesterol, high-density lipoprotein cholesterol, low-density lipoprotein cholesterol, glucose, sodium, potassium, chloride, calcium and inorganic phosphorus. Blood components were determined using conventional methods by Japan Clinical Laboratories, Inc. (Tokyo, Japan). Adverse events were assessed by the investigator at each study visit. Furthermore, subjects were requested to record the number of capsules ingested, subjective symptoms, any medications received and their meals for 3 days before the visit. The daily intakes of nutrients and purine were calculated by nutritionists from the food diary and pictures of meals ingested by the subjects for the 3 days before each visit.

Statistical analysis. Values are expressed as the mean \pm standard error. Baseline data of subjects, daily intakes of nutrients and safety data were compared by Dunnett's test among the 
placebo and the test supplement (at low and high doses of tuna extract) groups. In addition, the changes of serum uric acid were determined by Williams' test (15) among the placebo and the test supplement groups. Data were analyzed using R 3.2.2 scripting and statistical software (R Foundation for Statistical Computing, Vienna, Austria; https://www.r-project.org/). All the hypotheses were tested at the $5 \%$ level of significance. $\mathrm{P}<0.05$ was considered to indicate a statistically significant difference.

\section{Results}

Characteristics of the subjects. During the intervention, 1 subject in the placebo group withdrew from the study due to his own request. The remaining 47 subjects completed the study and were included in the statistical analysis for efficacy and safety. At the baseline, no significant differences were observed for age, physiological characteristics (body weight, body mass index, systolic blood pressure, diastolic blood pressure and pulse rate) and serum uric acid among the groups of placebo and the test supplement (at low and high doses of tuna extract) (Table II).

Effect of tuna extract ingestion on serum uric acid levels. Fig. 1 shows the changes of serum uric acid during the 4-week intervention and the subsequent 2 -week post-intervention follow-up. During the intervention, the uric acid levels in the test supplement (at low and high doses of tuna extract) groups were substantially decreased compared with the placebo group at weeks 2 and 4 . In addition, the uric acid levels in the test supplement group at a high-dose of tuna extract was significantly decreased compared with the placebo group at week 2 after the intervention (mean change from baseline: High-dose, $-0.49 \pm 0.19 \mathrm{mg} / \mathrm{dl}$; placebo, 0.14 $\pm 0.22 \mathrm{mg} / \mathrm{dl}$; $\mathrm{P}<0.05$ ).

The mean adherence rates were not different among the placebo and the test supplement groups $(95.7 \pm 0.5$, $95.9 \pm 0.8$ and $96.0 \pm 0.2 \%$ for the placebo, low and high dose, respectively). During and subsequent to the intervention, no significant differences were observed in the daily intakes of nutrients and purine between the placebo and the test supplement (at low and high doses of tuna extract) groups (Table III). Therefore, the oral administration of tuna extract was likely to decrease the serum uric acid in subjects with insignificantly high serum uric acid.

Adverse effects during regular tuna extract ingestion. During and subsequent to the intervention, 44 adverse events were reported in 5, 7 and 10 subjects receiving placebo, and test supplements at low and high doses, respectively. Relatively frequent adverse events reported were cold symptoms, gastric distress and headache. These events were generally mild and none were judged by the investigator to be associated with the study treatment. Furthermore, routine physical and cardiovascular characteristics, hematology and blood chemistry did not show any significant abnormalities during the intervention and follow-up periods in all the three groups (data not shown).

\section{Discussion}

The present study performed a randomized controlled study and investigated the effect of oral administration of the tuna
Table II. Baseline clinical characteristics of the study subjects.

\begin{tabular}{|c|c|c|c|}
\hline \multirow[b]{2}{*}{ Variables } & \multirow[b]{2}{*}{$\begin{array}{l}\text { Placebo } \\
(n=15)\end{array}$} & \multicolumn{2}{|c|}{$\begin{array}{l}\text { Test supplement } \\
\text { dose }\end{array}$} \\
\hline & & $\begin{array}{l}\text { Low } \\
(n=16)\end{array}$ & $\begin{array}{l}\text { High } \\
(n=16)\end{array}$ \\
\hline Age, years & $44.2 \pm 1.8$ & $45.0 \pm 1.9$ & $43.8 \pm 2.1$ \\
\hline Body weight, kg & $74.1 \pm 2.7$ & $74.8 \pm 2.0$ & $74.9 \pm 3.1$ \\
\hline Body mass index, $\mathrm{kg} / \mathrm{m}^{2}$ & $24.7 \pm 0.7$ & $25.5 \pm 0.7$ & $25.7 \pm 0.7$ \\
\hline $\begin{array}{l}\text { Systolic blood pressure, } \\
\mathrm{mmHg}\end{array}$ & $125.6 \pm 4.0$ & $124.3 \pm 3.8$ & $125.7 \pm 2.5$ \\
\hline $\begin{array}{l}\text { Diastolic blood pressure, } \\
\mathrm{mmHg}\end{array}$ & $79.5 \pm 2.6$ & $83.0 \pm 3.0$ & $77.9 \pm 2.0$ \\
\hline Pulse rate, beats $\min ^{-1}$ & $71.1 \pm 1.6$ & $70.1 \pm 1.4$ & $72.7 \pm 2.0$ \\
\hline Serum uric acid, mg/dl & $7.1 \pm 0.1$ & $7.2 \pm 0.1$ & $7.2 \pm 0.1$ \\
\hline
\end{tabular}

Values are expressed as the mean \pm standard error.

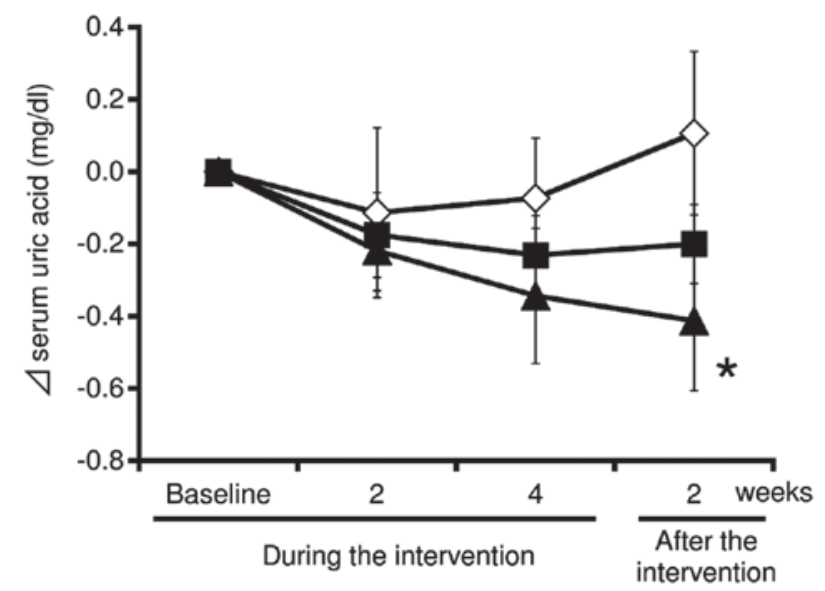

Figure 1. Changes of the serum uric acid in the subjects of the placebo and test supplement (at low and high doses of tuna extract) groups during and subsequent to the intervention. Serum uric acid levels were analyzed using serum samples collected from the subjects in the groups of placebo $(n=15$; open diamonds) and test supplements at low ( $\mathrm{n}=16$; closed squares) and high $(\mathrm{n}=16$; closed triangles) doses of tuna extract during and subsequent to the intervention. The absolute change from the baseline was calculated and plotted. The data are expressed as the mean \pm standard error. ${ }^{*} \mathrm{P}<0.05$ when compared with the placebo group.

extract (at low and high doses) on serum uric acid levels in gout-free subjects with insignificantly high serum uric acid. The results indicated that administration of the tuna extract containing the imidazole compounds resulted in a significant reduction in serum uric acid levels compared with administration of placebo. Moreover, there was a dose-dependent trend of uric acid reduction at week 4 during the intervention and week 2 after the intervention. Therefore, these results suggest that the oral administration of tuna extract containing the imidazole compounds exhibits a hypouricemic action on the individuals with insignificantly high serum uric acid.

Only a limited number of studies have investigated the hypouricemic effect of the imidazole compounds. The study by Noguchi et al (16) fed a diet containing $1 \%$ inosine and 
Table III. Mean daily intakes of nutrients and purine for 3 days before the bi-weekly blood collection.

\begin{tabular}{|c|c|c|c|c|}
\hline \multirow[b]{2}{*}{ Nutrients } & \multicolumn{3}{|c|}{ During the intervention } & \multirow{2}{*}{$\begin{array}{c}\begin{array}{c}\text { After the } \\
\text { intervention }\end{array} \\
\text { Week } 2\end{array}$} \\
\hline & Baseline & Week 2 & Week 4 & \\
\hline \multicolumn{5}{|c|}{ Energy, kj/day } \\
\hline Placebo & $7,976 \pm 346$ & $8,541 \pm 550$ & $8,831 \pm 446$ & $8,393 \pm 552$ \\
\hline Low dose & $8,855 \pm 353$ & $9,256 \pm 469$ & $9,529 \pm 486$ & $9,072 \pm 456$ \\
\hline High dose & $7,568 \pm 333$ & $7,978 \pm 412$ & $8,705 \pm 510$ & $8,070 \pm 487$ \\
\hline \multicolumn{5}{|c|}{ Protein, g/day } \\
\hline Placebo & $65.4 \pm 3.7$ & $71.0 \pm 5.3$ & $73.9 \pm 3.7$ & $71.1 \pm 4.1$ \\
\hline Low dose & $70.5 \pm 3.3$ & $76.1 \pm 5.3$ & $76.8 \pm 4.6$ & $76.1 \pm 4.4$ \\
\hline High dose & $68.5 \pm 4.7$ & $65.9 \pm 4.1$ & $72.2 \pm 4.2$ & $70.8 \pm 4.9$ \\
\hline \multicolumn{5}{|l|}{ Fat, g/day } \\
\hline Placebo & $63.6 \pm 5.1$ & $62.3 \pm 5.8$ & $71.7 \pm 5.8$ & $67.3 \pm 5.7$ \\
\hline Low dose & $72.6 \pm 3.0$ & $80.4 \pm 4.3$ & $85.7 \pm 5.9$ & $78.1 \pm 3.8$ \\
\hline High dose & $57.7 \pm 4.6$ & $68.2 \pm 7.4$ & $77.2 \pm 6.4$ & $71.2 \pm 8.4$ \\
\hline \multicolumn{5}{|c|}{ Carbohydrate, g/day } \\
\hline Placebo & $256.0 \pm 12.0$ & $286.8 \pm 17.7$ & $279.4 \pm 15.1$ & $266.3 \pm 17.6$ \\
\hline Low dose & $284.0 \pm 15.4$ & $284.8 \pm 15.9$ & $287.3 \pm 16.8$ & $278.0 \pm 16.5$ \\
\hline High dose & $243.3 \pm 11.2$ & $247.1 \pm 9.9$ & $262.8 \pm 17.0$ & $240.8 \pm 13.4$ \\
\hline \multicolumn{5}{|c|}{ Purine, g/day } \\
\hline Placebo & $276.1 \pm 20.6$ & $296.8 \pm 28.1$ & $307.8 \pm 20.3$ & $302.0 \pm 23.1$ \\
\hline Low dose & $259.6 \pm 13.2$ & $306.9 \pm 32.0$ & $301.8 \pm 24.7$ & $320.2 \pm 34.7$ \\
\hline High dose & $296.1 \pm 22.9$ & $265.3 \pm 20.9$ & $292.0 \pm 19.1$ & $276.6 \pm 19.8$ \\
\hline
\end{tabular}

Values are expressed as the mean \pm standard error.

$1 \%$ anserine to Wistar rats (male, 7 weeks old) ad libitum. After 1 week of administration, the serum uric acid levels in the rats in the inosine with anserine group were lower compared to those in the inosine group (as a control). Microarray analysis showed a marked increase in the expression of hypoxanthine phosphoribosyltransferase and lactate dehydrogenase in the livers of the rats in the inosine with anserine group compared with those in the inosine group. Hypoxanthine phosphoribosyltransferase salvages hypoxanthine and guanine to renew purine synthesis (17), resulting in the inhibition of uric acid synthesis by the tuna extract containing anserine. Lactate dehydrogenase catalyzes the interconversion of pyruvate and lactate. Lactate directly activates urate transporter 1 (URAT1), contrary to several uricosurics. URAT1 is the most important element in the mechanism involved in the reabsorption and urinary excretion of uric acid (18). In addition, ingested anserine is absorbed intact in human blood, and is hydrolyzed to $\pi$-methylhistidine and $\beta$-alanine by serum and tissue carnosinases for $4 \mathrm{~h}$ after ingestion (19). Intact anserine and $\pi$-methylhistidine ( $8 \%$ anserine and $82 \% \pi$-methylhistidine of the ingested amount of anserine) are excreted into the urine (20). Therefore, anserine and $\pi$-methylhistidine, with their proton-buffering capacity, may stabilize the $\mathrm{pH}$ of urine to improve the solubility of uric acid and accelerate excretion, similar to the mechanism of sodium potassium citrate (21). These hypotheses require further investigation. Moreover, although almost all the anserine is excreted into the urine over the $24 \mathrm{~h}$ after ingestion, the serum uric acid levels were reduced at week 2 after the intervention. This phenomenon cannot be accounted for by the aforementioned hypotheses alone. Baguet et al (22) reported changes in the carnosine content in human skeletal muscles subsequent to $\beta$-alanine ingestion. In the present study, muscle carnosine increased and returned to baseline values within 9 weeks. Therefore, carnosine was synthesized by $\beta$-alanine-derived anserine, and may be correlated with the hypouricemic effect.

Previous studies have supported the safety of anserine and carnosine ingestion. Chicken breast extract (CBEX) containing $\geq 35 \%$ anserine and carnosine did not result in mutagenicity, assessed using the Ames assay, and no adverse effects were observed in a 90-day chronic toxicity test $(2,000 \mathrm{mg} / \mathrm{kg} / \mathrm{day})$ in either male or female rats (23). Similarly, in humans, no adverse events or abnormal changes were observed in the blood examination, urinalysis or physical examination after regular ingestion of CBEX (1,200 mg/day of anserine and carnosine) for 4 weeks (24). In the present study, no severe adverse events or marked changes were observed in the general condition of the patients, hematology or blood biochemistry during regular ingestion of the tuna extract. Therefore, continuous administration of the tuna extract to subjects with insignificantly high serum uric acid is not likely to cause adverse health effects. 
In conclusion, these results suggest that the tuna extract may be a beneficial and safe food ingredient for individuals with insignificantly high serum uric acid. Since a number of studies have shown antihypertensive (25) and hypoglycemic effects (26) of anserine, its ingestion results in overall health benefits to individuals. However, although no significant differences were observed between the tuna extract and placebo groups during the intervention, a significant difference was identified following the intervention; this could be due to the small sample size, insufficient study duration or dose tested. Therefore, additional studies are required for more accurate assessment of the efficacy of the tuna extract. Moreover, further studies on the evaluation of uric acid excretion into the urine are required to evaluate the mechanism of serum uric acid level reduction instigated by tuna extract ingestion, including the imidazole compounds contribution.

\section{Acknowledgements}

The authors would like to thank Dr Shin Fujimori and Dr Kiyoko Kaneko (Teikyo University) for their assistance in discussions regarding the results. Furthermore, the authors also thank Ms. Fumiyo Ohya-Nakano for her assistance in the validation of analysis and study preparation. The authors would like to thank Enago (www.enago.jp) for the English language review.

\section{References}

1. Choi HK and Ford ES: Prevalence of the metabolic syndrome in individuals with hyperuricemia. Am J Med 120: 442-447, 2007.

2. Wortmann RL: Gout and hyperuricemia. Curr Opin Rheumatol 14: 281-286, 2002.

3. Ishizaka N, Ishizaka Y, Toda E, Nagai R and Yamakado M: Association between serum uric acid, metabolic syndrome, and carotid atherosclerosis in Japanese individuals. Arterioscler Thromb Vasc Biol 25: 1038-1044, 2005.

4. Shoji A, Yamanaka H and Kamatani N: A retrospective study of the relationship between serum urate level and recurrent attacks of gouty arthritis: Evidence for reduction of recurrent gouty arthritis with antihyperuricemic therapy. Arthritis Rheum 51: 321-325, 2004.

5. Roujeau JC, Kelly JP, Naldi L, Rzany B, Stern RS, Anderson T, Auquier A, Bastuji-Garin S, Correia O, Locati F, et al: Medication use and the risk of Stevens-Johnson syndrome or toxic epidermal necrolysis. N Engl J Med 333: 1600-1607, 1995.

6. Hande KR, Noone RM and Stone WJ: Severe allopurinol toxicity. Description and guidelines for prevention in patients with renal insufficiency. Am J Med 76: 47-56, 1984.

7. Yamanaka H, Ueda T, Ohno I: Guideline for the management of hyperuricemia and gout. 2nd edition. Medical Review Co., Ltd., Tokyo, 2010.

8. Kohen R, Yamamoto Y, Cundy KC and Ames BN: Antioxidant activity of carnosine, homocarnosine, and anserine present in muscle and brain. Proc Natl Acad Sci USA 85: 3175-3179, 1988.
9. Abe H: Role of histidine-related compounds as intracellular proton buffering constituents in vertebrate muscle. Biochemistry (Mosc) 65: 757-765, 2000.

10. Boldyrev A, Bulygina E, Leinsoo T, Petrushanko I, Tsubone S and Abe $\mathrm{H}$ : Protection of neuronal cells against reactive oxygen species by carnosine and related compounds. Comp Biochem Physiol B Biochem Mol Biol 137: 81-88, 2004.

11. Severin SE, Skolysheva LK, Shur SA and Vulfson PL: The $\mathrm{pH}$-dependent conformational transition in glycogen phosphorylase $b$. The effect of carnosine and anserine on its activity. Biochem Int 20: 227-238, 1990.

12. Ikeda T, Kimura K, Hama T and Tamaki N: Activation of rabbit muscle fructose 1,6-bisphosphatase by histidine and carnosine. J Biochem 87: 179-185, 1980.

13. Chang WTH: Composition containing dipeptide of histidine and alanine for reducing uric acid and method for reducing uric acid using the dipeptide. US Patent 7498301. Filed December 22, 2006; issued March 3, 2009.

14. Abe $\mathrm{H}$ and Ohmama S: Effect of starvation and sea-water acclimation on the concentration of free l-histidine and related dipeptides in the muscle of eel, rainbow trout and Japanese dace. Comp Biochem Physiol Part B Comp Biochem 88: 507-511, 1987.

15. Williams DA: A test for differences between treatment means when several dose levels are compared with a zero dose control. Biometrics 27: 103-117, 1971.

16. Noguchi Y, Shimizu J, Mano H, Matahira Y and Wada M: Effect of anserine administration on purine metabolism. In: Proceedings of 60th Annual Meeting of Japan Society of Nutrition and Food Science, Tokyo, p268, 2006.

17. Curto R, Voit EO and Cascante M: Analysis of abnormalities in purine metabolism leading to gout and to neurological dysfunctions in man. Biochem J 329 (Pt 3): 477-487, 1998.

18. Enomoto A, Kimura H, Chairoungdua A, Shigeta Y, Jutabha P, Cha SH, Hosoyamada M, Takeda M, Sekine T, Igarashi T, et al: Molecular identification of a renal urate anion exchanger that regulates blood urate levels. Nature 417: 447-452, 2002.

19. Kubomura D, Matahira Y, Masui A and Matsuda H: Intestinal absorption and blood clearance of L-histidine-related compounds after ingestion of anserine in humans and comparison to anserine-containing diets. J Agric Food Chem 57: 1781-1785, 2009.

20. Abe H, Okuma E, Sekine H, Maeda A and Yoshiue S: Human urinary excretion of L-histidine-related compounds after ingestion of several meats and fish muscle. Int J Biochem 25: 1245-1249, 1993.

21. Kessler T and Hesse A: Cross-over study of the influence of bicarbonate-rich mineral water on urinary composition in comparison with sodium potassium citrate in healthy male subjects. Br J Nutr 84: 865-871, 2000.

22. Baguet A, Reyngoudt H, Pottier A, Everaert I, Callens S, Achten E and Derave W: Carnosine loading and washout in human skeletal muscles. J Appl Physiol 1985 106: 837-842, 2009.

23. Sato M, Karasawa N, Shimizu M, Morimatsu F and Yamada R: Safety evaluation of chicken breast extract containing carnosine and anserine. Food Chem Toxicol 46: 480-489, 2008.

24. Aoyagi S, Sugino T, Kajimoto Y and Nishitani M: Safety of excess administration of CBEX-Dr-containing drink on healthy people. Jpn Pharmacol Ther 36: 199-212, 2008.

25. Tanida M, Shen J, Kubomura D and Nagai K: Effects of anserine on the renal sympathetic nerve activity and blood pressure in urethane-anesthetized rats. Physiol Res 59: 177-185, 2010.

26. Kubomura D, Matahira Y, Nagai K and Niijima A: Effect of anserine ingestion on hyperglycemia and the autonomic nerves in rats and humans. Nutr Neurosci 13: 183-188, 2010. 